\title{
Análise e caracterização de modelos de custos que utilizam o valor de Shapley para alocação de custos entre departamentos
}

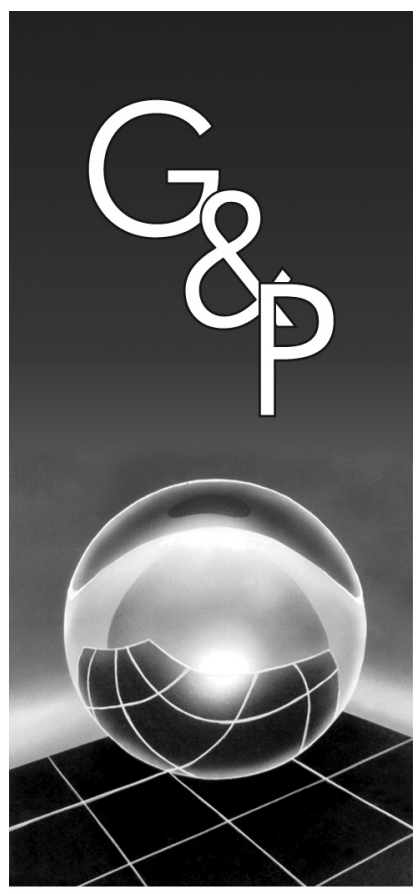

\author{
Francisco Antonio Bezerra \\ Jefferson Fernando Grande \\ Adriano José da Silva
}

Resumo

Visando obter benefícios (economias de escala e escopo), diversas organizações criam estruturas de prestação de serviços internos. Os departamentos de informática são exemplos disso. A distribuição dos custos de prestação de serviços internos entre os departamentos usuários destes serviços promove o controle dos custos e incentiva a melhoria da eficiência na utilização dos recursos. Este trabalho descreve as características de um modelo de alocação dos custos de prestação de serviços internos baseado nos conceitos da teoria dos jogos cooperativos. Foi utilizado o valor de Shapley para se encontrar a solução do jogo cooperativo. É demonstrado que este modelo matemático de distribuição de ganhos (economias de escala e escopo) produz resultados que, sob a ótica do tomador de decisão racional, gera o melhor resultado de distribuição de custos, pois está baseado no custo marginal de inserção de cada um dos usuários do serviço interno. Em termos metodológicos, esta pesquisa pode ser classificada, quanto ao seu objetivo, como sendo uma pesquisa exploratória e, quanto ao seu delineamento, trata-se de um levantamento bibliográfico.

Palavras-Chave: Alocação de custos. Teoria dos jogos cooperativos. Valor de Shapley.

\section{Introdução}

A alocação de custos é o processo de atribuição de custos quando não é possível determinar o consumo efetivo de determinado recurso (pessoal, depreciação etc.) por um objeto de custo específico. Neste caso, na alocação de custos, sempre são utilizados critérios alternativos que são considerados uma aproximação da forma como um objeto de custo consome um recurso. $\mathrm{O}$ objeto de custo neste artigo representa tudo aquilo de que se deseja saber o custo (DRURY, 2004).

Parte integrante de um sistema de alocação de custos, os sistemas de acumulação associam custos aos departamentos de uma organização, tal sistema vai sofrendo movimentações durante um período de tempo à medida que estes departamentos vão consumindo recursos. Os valores dos custos, ao final de um período, servem para avaliar a performance do departamento, ao mesmo tempo que critérios de associação permitem a distribuição destes custos para os produtos. Para Kaplan e Atkinson (1998):

Um sistema de custos tradicional possui uma estrutura de dois estágios [...]. No primeiro estágio, os departamentos de serviço são distribuídos entre os departamentos operacionais ou de produção. [...]. No segundo estágio [...], os custos são distribuídos para os produtos processados por estes departamentos. (KAPLAN; ATKINSON, 1998).

Neste artigo, propõe-se um modelo de distribuição de custos para o primeiro estágio de alocação de custos. Diferentemente da grande maioria dos trabalhos na área de custos, este não apresenta uma solução para a distribuição de custos aos produtos (segundo estágio de alocação de custos). Já existem diversos trabalhos veiculados em importantes publicações na área contábil, que utilizam o mesmo referencial teórico inserido neste artigo para realizar a última etapa de distribuição de custos (HAMLEN; HAMLEN; TSCHIRHART, 1977; BALACHANDRAN; RAMAKRISHNAN, 1981; CALLEN, 1978; BUTLER; WILLIAMS, 2002). Essa, aliás, é uma das motivações deste trabalho, pois, no entender dos autores, a utilização dos conceitos da teoria dos jogos foi muito pouco explorada quanto aos aspectos 
de aplicação desses conceitos na distribuição dos custos ainda na primeira fase de alocação. A sua principal aplicação tem sido na distribuição de custos (comuns) aos produtos.

Conforme Kaplan e Atkinson (1998), a distribuição dos custos de prestação de serviços internos entre os departamentos usuários (operacionais ou não) promove o controle dos custos e incentiva a melhoria da eficiência na utilização dos recursos, visto que:

a) incentiva a melhoria da eficiência na utilização dos recursos dos gestores dos departamentos de serviço; e

b) motiva a prudente utilização dos serviços internos por parte dos departamentos usuários.

Para Zimmerman (2003), existem três razões para a locação dos custos dos departamentos de serviços:

a) a precificação de um serviço interno (mesmo que pelo custo) auxilia na utilização de recursos escassos. Com preço zero, a demanda será quase sempre maior que a oferta do serviço;

b) pela alocação dos custos dos serviços internos, os gestores da companhia receberão a informação da demanda total pelo serviço pela alocação dos custos aos departamentos usuários. Isto auxilia na determinação da otimização da escala do departamento prestador do serviço que pode dimensionar melhor sua estrutura; e

c) a comparação entre a alocação de custos entre departamentos de serviços e departamentos usuários e o preço de um prestador externo do mesmo serviço possibilita medir a eficiência operacional dos departamentos de serviços internos.

Não é objetivo deste trabalho discutir questões relacionadas ao estabelecimento de preços de transferência. Assume-se que a mensagem relevante para controle dos custos pode ser obtida pela simples transferência dos custos incorridos na prestação do serviço, sem a inclusão de margens de lucros para os departamentos de serviços.

Nas palavras de Kaplan e Atkinson (1998):

A distribuição dos custos dos departamentos de serviços internos para os departamentos de produção, com o propósito de controle dos custos e da eficiência na utilização de recursos, requer a criação de uma medida bem acurada do consumo dos serviços internos para cada departamento. Estimativas e alocações de custos podem ser utilizadas para o custeio dos produtos e avaliação dos estoques, mas eles não são úteis quando os gestores estão focados no controle de produção e no uso de recursos comuns. Isto porque a distribuição será baseada numa medida não relacionada à demanda de produção feita para os departamentos de serviços. Os custos alocados não são resultado das ações tomadas pelos gestores ou funcionários do departamento de produção. Sendo assim, a mensagem proporcionada pela alocação dos custos não será um feedback útil na avaliação da performance operacional do departamento de produção durante um período. (KAPLAN; ATKINSON, 1998).

Assim, este trabalho trata da modelagem de um mecanismo de alocação dos custos de prestação de serviços internos aos departamentos usuários do serviço.

No entanto, cria-se um modelo que carrega uma mensagem, pelo critério de alocação adotado, que pode ser utilizada como feedback na avaliação da performance do departamento usuário do serviço interno, pois está baseado no custo marginal que o departamento usuário causou na prestação do serviço interno.

O questionamento que motiva a realização deste trabalho é o seguinte: Como as propriedades que delineiam o modelo de alocação de custos dos departamentos de serviços internos baseado na teoria dos jogos cooperativos devem ser entendidas e utilizadas para tomada de decisão?

Desta forma, o objetivo deste trabalho é apresentar as diferenças e descrever as características de um modelo de alocação de custos entre departamentos que, baseado nos conceitos da teoria dos jogos cooperativos, possa ser utilizado como um instrumento na tomada de decisão e no controle dos custos de prestação de serviços internos.

Em termos metodológicos, esta pesquisa pode ser classificada, quanto ao seu objetivo, como sendo uma pesquisa exploratória e, quanto ao delineamento, pode-se afirmar que corresponde a um levantamento bibliográfico, pois foi desenvolvida a "partir de material já elaborado, constituído principalmente de livros e artigos científicos" (GIL, 1987).

\section{Teoria dos jogos cooperativos na alocação de custos}

A teoria dos jogos cooperativos pressupõe a existência de uma estrutura comum que, ao ser utilizada por duas ou mais entidades (jogadores), gera algum tipo de benefício. Esse benefício (ganho) deve ser distribuído entre as partes envolvidas de acordo com alguns critérios de distribuição (axiomas). Jensen (1977), Hamlen et al. (1977), Balachandran e Ramakrishnan (1981) são exemplos de trabalhos que utilizaram a teoria dos jogos cooperativos para alocação de custos.

Tome-se, como exemplo, uma empresa que centraliza as atividades de Tecnologia de Informação (TI) em um único departamento por acreditar que isso seja mais barato do que se cada departamento possuísse sua própria estrutura de TI. A mesma análise pode ser feita, por exemplo, na centralização das atividades dos departamentos de compras, recursos humanos, contabilidade etc. 
No entanto, ao tomar essas decisões de centralização, as empresas defrontam-se com um problema: como alocar o custo pelos departamentos centralizadores para os departamentos usuários do serviço?

A teoria dos jogos cooperativos possui um arcabouço teórico que pode ser utilizado pelos gestores departamentais que, agindo de forma racional com o foco estritamente voltado para a maximização do seu resultado, decidem como os custos de estruturas comuns podem ser distribuídos entre os usuários do serviço.

Em primeiro lugar é necessário compreender que a existência de estruturas centralizadas dentro das organizações tem uma justificativa muito simples: ou tem-se uma situação na qual existe ganho de escopo ou de escala, ou ainda os dois. A economia de custo conseguida pela implantação de atividades centralizadas que prestam serviços para todos os departamentos da empresa deveria representar um ganho que precisa ser distribuído entre os usuários do serviço, pois não seria realizada a centralização se não ocorresse ganho algum para a organização, tais como redução de custos e de risco.

\subsection{Ganho de escopo}

Panzar e Willig (1981) definiram o termo economia de escopo para descrever uma condição na qual é mais barato combinar dois ou mais produtos em uma única fábrica do que produzi-los separadamente.

Se existe economia de escopo em uma organização, existe também uma função de custos subaditiva, que estabelece que o fornecimento de recursos comuns custa menos do que o fornecimento desses mesmos recursos individualmente para cada usuário.

Para deixar mais claro o que vem a ser uma função de custos subaditiva, suponha que existam $n$ departamentos $\left(d_{1}, d_{2}, \ldots, d_{n}\right)$ e que todos utilizem serviços diferenciados entre si $d_{1}, d_{2}, \ldots, d_{n}$ prestados por um departamento de serviço interno. A função de custo de cada um dos departamentos é dada por $C_{1}\left(d_{1}\right), C_{2}\left(d_{2}\right), \ldots, \mathrm{C}_{n}\left(d_{n}\right)$.

A economia de escopo ocorre quando o custo de recursos comuns é estritamente subaditivo, ou seja, $C_{N}\left(d_{1}, d_{2}, \ldots, d_{n}\right)<\Sigma C_{i}\left(d_{i}\right)$, em que $i=1, \ldots, n$ e $C_{N}\left(d_{1}\right.$, $\left.d_{2}, \ldots, d_{n}\right)$ é o custo total dos recursos comuns. Em outras palavras, a economia de escopo ocorre quando o custo total do departamento de serviços internos for menor do que o somatório do custo da descentralização da atividade, considerando-se todos os departamentos usuários.

Quando a economia de escopo existe, um benefício (economia de custo) surge pela criação de estruturas comuns que serão utilizadas por todos dentro da empresa. A economia de custo pode ser descrita da seguinte forma: $\sum C_{i}\left(d_{i}\right)-C_{N}\left(d_{1}, d_{2}, \ldots, d_{n}\right)$. Simplificando um pouco, no caso específico de dois departamentos usuários, pode-se descrever a economia de custo pela presença de economia de escopo da seguinte forma: $C_{1}\left(d_{1}\right)+C_{2}\left(d_{2}\right)-C_{12}\left(d_{1}, d_{2}\right)$.
Se, por outro lado, fosse provável a decomposição da função de custos subaditiva, $C_{12}\left(d_{1}, d_{2}\right)$, seria possível obter o custo efetivo da prestação de serviço para cada usuário interno. Ou seja, caso se conseguisse segregar $C_{12}\left(d_{1}, d_{2}\right)$ em $C^{*}{ }_{1}\left(d_{1}\right)+C^{*}{ }_{2}\left(d_{2}\right)$, isso resultaria em $C^{*}{ }_{1}\left(d_{1}\right)$ e $C^{*}{ }_{2}\left(d_{2}\right)$, que representam os custos que devem ser "ressarcidos" para o departamento de serviço interno.

\subsection{Custo fixo}

A economia de escopo ocorre pelo fato dos recursos utilizados na produção de produtos/serviços possuírem características que dão origem às funções de custos subaditivas. Entre estas características estão: os custos fixos e o efeito causado pelo ganho de escala.

Um dos motivos da existência da economia de custo obtida pela centralização de alguns serviços internos é fruto da divisão imperfeita de determinados recursos utilizados na disponibilização dos serviços internos. Esses recursos, segundo Bailey e Friedlaender (1982), permitem o consumo da capacidade não utilizada (CNU). Como exemplos desse tipo de recurso, podem ser citados os equipamentos utilizados para a realização dos serviços internos, a mão-de-obra envolvida em diversas atividades consumidas por dois ou mais serviços internos, dentre outros.

Os recursos categorizados como custos fixos são, em geral, comprometidos e adquiridos em intervalos antes da efetiva utilização. No momento de sua aquisição, não se sabe se esse recurso irá ser consumido completamente. Caso haja uma utilização abaixo do esperado, tem-se, então, a presença de capacidade não utilizada que precisa ser reduzida ou eliminada.

Para Horngreen (1986), os custos fixos, custos de capacidade ou capacity costs:

\section{Refletem a capacidade de sustentar o volume de atividade planejado. Uma vez adquirida, a capa- cidade deve ser utilizada ao máximo, desde que, evidentemente, o aumento de receita exceda o aumento dos custos variáveis à medida que aumenta o volume. (HORNGREEN, 1986).}

Em poucas palavras, os custo fixos têm duas implicações mais importantes para a administração. Primeiramente, o planejamento é crucial. Em segundo lugar, a total utilização da capacidade é frequentemente desejável.

Os custos fixos são representados por funções de custos intervalares (LEIKAM, 2000). Assuma-se que o recurso $j$ é um custo fixo utilizado para produção do serviço interno $i$. Um intervalo do recurso ( $j$ ) é representado por $I_{j}$, que indica a quantidade fixa de recurso $j$ (ou capacidade do recurso $j$ ) que deve ser comprada, levando-se em consideração o atual nível de consumo do serviço $i$. Assuma-se também que o número de intervalos a serem adquiridos do recurso $j$, necessários para atender à demanda pelos 
serviços internos é $N_{i j}$. A quantidade total do recurso $j$ é igual a $I_{j} N_{i j}$. Suponha-se que $d_{\mathrm{ij}}$ representa a demanda do departamento $D_{\mathrm{i}}$ do recurso $j$, na qual a capacidade não utilizada é calculada pela expressão $I_{j} N_{i}-d_{i j}$.

O custo do serviço $i$ é dado pela seguinte função de custo $C_{i}=\sum P_{j} N_{i j}$, em que:

a) $C_{i}$ é o custo do serviço $i$;

b) $P_{j}$ é o preço pago pela aquisição da capacidade do recurso $j$; e

c) $N_{i j}$ é o número de intervalos do recurso $j$ necessários para atender à demanda do serviço $i$.

Para assimilação dos conceitos descritos, em uma situação de economia de escopo, considere-se o exemplo a seguir, que foi adaptado de Leikam (2000). Admita-se que a capacidade de armazenamento disponível para a prestação do serviço "armazenamento de dados", fornecido pelo departamento de TI, é de $500 \mathrm{Mb}$ por um custo de $\$ 5.000,00$ (Tabela 1 ).

A empresa tem duas opções com relação à disponibilização dos recursos para os departamentos (1, 2 e 3): permitir que cada departamento compre os recursos necessários para suas atividades (serviços descentralizados) ou centralizar a gestão dos recursos em um quarto departamento, no caso, o de TI.

No caso da empresa permitir que os departamentos adquiram individualmente os recursos de TI, tem-se um aproveitamento inferior ao que os recursos são capazes de atender. O departamento 1, por exemplo, tem uma demanda de memória de $945 \mathrm{Mb}$. Caso venha a adquirir sozinho esse recurso, deverá dispor de duas memórias de $500 \mathrm{Mb}$. Significa que o departamento 1 não estará utilizando $55 \mathrm{Mb}$. A mesma situação repete-se para os outros dois departamentos. Contudo, caso a empresa decida centralizar a gestão dos recursos de TI, as demandas podem ser analisadas e atendidas de forma conjunta, permitindo que a utilização dos recursos seja otimizada e que não haja desperdício.

Nesse exemplo, a CNU foi completamente eliminada pela centralização dos serviços consumidos pelos departamentos 1, 2 e 3 no departamento de TI. Pode-se concluir

Tabela 1. Análise de demanda de armazenamento.

\begin{tabular}{lccccc}
\hline \multicolumn{1}{c}{$\begin{array}{c}\text { Recurso: } \\
\text { memória }\end{array}$} & & $\begin{array}{c}\text { Serviços desconhecidos } \\
\text { (usuários) }\end{array}$ & $\begin{array}{c}\text { Centralizado } \\
\text { TI }\end{array}$ \\
\hline $\begin{array}{l}I_{\mathrm{j}}=500 \mathrm{Mb} \\
P_{\mathrm{j}}=5.000,00\end{array}$ & & 1 & 2 & 3 & 123 \\
$\begin{array}{l}\text { Memória } \\
\text { necessária }\end{array}$ & $d_{i j}$ & 945 & 1.050 & 1.505 & 3.500 \\
$\begin{array}{l}\text { Número de } \\
\text { intervalos }\end{array}$ & $N_{i j}$ & 2 & 3 & 4 & 7 \\
$\begin{array}{l}\text { Capacidade } \\
\text { prática }\end{array}$ & $I_{j} N_{i j}$ & 1.000 & 1.500 & 2.000 & 3.500 \\
CNU & $I_{\mathrm{j}} N_{\mathrm{ij}}-d_{i j}$ & 55 & 450 & 495 & - \\
\hline
\end{tabular}

Fonte: adaptado de Leikam (2000). que a economia de escopo tende a ocorrer quando existe uma parcela da capacidade adquirida de recursos fixos que não está sendo utilizada. Dessa forma, tem-se que $d_{i j}<I_{j} N_{i j}$, ou $\frac{d_{i j}}{I_{j}}<N_{i j}$.

Quando se tem um departamento utilizando o mesmo recurso fixo para a realização de diversos serviços internos, a economia de custo aparece quando a CNU é reduzida ou eliminada.

\subsection{Efeitos da economia de escala}

Outro fator responsável pelo surgimento da economia de escopo, já que corresponde a recursos com função de custos subaditiva, são os recursos que sofrem os efeitos da economia de escala (BAILEY; FRIEDLAENDER, 1982).

Quando os efeitos da economia de escala existem, o custo unitário médio de um recurso comum a diversos serviços é decrescente, o que indica a presença de função de custo subaditiva. Se as empresas utilizarem recursos comuns com essas características em suas funções de custos, economias de custos surgirão pela centralização do consumo desses recursos em departamentos prestadores de serviços internos.

Para os recursos que sofrem efeitos de economia de escala, a função de custos está relacionada com as unidades produzidas, ou seja: $C_{i}=\sum_{j} C\left(d_{i j}\right)$.

A economia de custo, nos casos em que os recursos sofrem os efeitos dos ganhos de escala, é devida à redução do custo médio do recurso por unidade. A possibilidade de economia é função da demanda total e é definida como sendo: $\sum_{i} C\left(d_{i j}\right)-C\left(\sum_{i} d_{i j}\right)$.

Admita-se que determinado serviço, prestado pelo departamento de TI para um usuário em especial, necessite de certa quantidade de minutos de CPU para ser concluído. Suponha-se que esse recurso possa ser aproveitado para a prestação de outros serviços dentro da empresa. Sendo assim, pode-se criar a seguinte situação (Tabela 2):

Pode-se perceber que este é um tipo de recurso cujo custo varia em função do número de unidades produzidas. Se o departamento 1 adquirisse a quantidade que precisa,

Tabela 2. Análise de demanda de tempo de CPU.

\begin{tabular}{lccccc}
\hline \multicolumn{1}{c}{$\begin{array}{c}\text { Recurso: } \\
\text { tempo de CPU }\end{array}$} & \multicolumn{4}{c}{$\begin{array}{c}\text { Serviços descentralizados } \\
\text { (usuários) }\end{array}$} & $\begin{array}{c}\text { Centralizado } \\
\text { TI }\end{array}$ \\
\hline & & 1 & 2 & 3 & 123 \\
Tempo de CPU & $d_{i j}$ & 3.960 & 6.600 & 22.440 & 33.000 \\
necessário & & & & & \\
Custo & $C\left(d_{i j}\right)$ & 31.680 & 39.600 & 89.760 & 115.500 \\
$\begin{array}{l}\text { Custo médio/ } \\
\text { tempo de CPU }\end{array}$ & $C\left(d_{i j}\right) / d_{i j}$ & 8,00 & 6,00 & 4,00 & 3,50 \\
\hline
\end{tabular}

Fonte: adaptado de Leikam (2000). 
estaria pagando $\$ 8,00$ unidades monetárias pelo tempo de CPU que necessita. Contudo, se o departamento de TI fizer esta aquisição conjunta para os três departamentos, o custo unitário de aquisição seria de $\$ 3,50$. Para esse tipo de recurso não existe a presença de CNU.

Sabe-se que o conjunto dos recursos utilizados pelos departamentos prestadores de serviço (custo total) possui características que podem gerar economias de custo pela centralização (existência de custos fixos e efeitos sofridos pelo ganho de escala). A função de custo do departamento de serviço interno é uma mescla de recursos com uma ou outra característica (ou as duas). O que se precisa descobrir é uma forma de decompor o custo para cada serviço prestado pelos departamentos de serviços internos, para que esse valor sirva de base para alocação de custos entre os departamentos.

\subsection{Função característica e a alocação de custos}

Shubik (1962) foi o primeiro autor a descrever como poderiam ser utilizados os conceitos da teoria dos jogos, em especial o valor de Shapley, em diversas situações nas quais surgem ganhos em decorrência da coalizão de dois ou mais departamentos ou unidades de negócio de uma empresa.

O artigo de Shubik (1962) teve por objetivo descrever como os conceitos (axiomas) da teoria dos jogos cooperativos poderiam ser utilizados para alocação de custos conjuntos (Joint Costs). Segundo Shubik (ibid.):

A teoria dos jogos cooperativos, desenvolvida por Von Neumann e Morgenstern, depende de uma medida de relacionamento que é maior em situações de ganhos obtidos por um grupo de indivíduos que está disposto a agir em conjunto, quando comparado com as ações individuais. $\mathrm{O}$ resultado de uma corporação pode ser enxergado como dependente da soma dos ganhos conjuntos que podem ser obtidos pela coordenação ótima de todos os negócios da empresa. [...] Os jogadores no jogo descrito por von Neumann e Morgenstern podem ser considerados como os ramos de negócios ou os departamentos da empresa ou mesmo as seções de uma fábrica. (SHUBIK, 1962).

O autor (ibid.) ainda comenta que a medida de "complementaridade" é dada pela função característica, e afirma que:

A função característica é chamada de superaditiva porque o ganho obtido por qualquer grupo de participantes é sempre igual ou maior do que pode ser obtido pelas ações individuais. Por exemplo, um casaco é melhor do que duas metades de um mesmo casaco. A função característica é um caminho que permite que a complementaridade possa ser descrita entre diferentes objetos ou grupos. (SHUBIK, 1962).
A função característica atribui um valor a uma combinação de jogadores que decidiram realizar uma coalizão (trabalhar em conjunto ou cooperarem entre si). $\mathrm{O}$ valor atribuído pela função característica à coalizão representa o máximo (de custo ou receita) a ser atribuído à coalizão de jogadores, independentemente do que façam os demais jogadores que não estão participando da coalizão.

A função característica é representada por $v(S)$, valor total obtido pela coalizão $S$, sendo que $S \subset N$. No caso dos jogos cooperativos, a função característica deve satisfazer às seguintes condições:

a) $v(\varnothing)=0$; e

b) $v(R \cup S) \geq v(R)+v(S)$, sendo $R \cap S=\varnothing$.

A primeira condição descreve que uma coalizão sem jogadores não gera ganho (ou economia de custo). A segunda condição indica que uma coalizão de $R \operatorname{com} S$ pode obter, no mínimo, a soma dos seus ganhos, caso ajam isoladamente, ou um valor maior com a coalizão, nunca menor (se fosse menor os jogadores não aceitariam participar da coalizão).

Os benefícios conseguidos pela coalizão $S$ são calculados pela expressão $\sum v(i)-v(S)$, em que $v(i)$ representa o valor do jogador $i$ agindo isoladamente. Todavia, sabe-se que os benefícios gerados pela coalizão $S$ precisam ser distribuídos entre os jogadores que compõem a coalizão. A alocação do benefício será representada por $x_{i}$, em que $i \in S \subset N$ e o conjunto de alocações racionais é um vetor de ganhos que será definido como $x=\left(x_{1}, x_{2}, \ldots, x_{n}\right)$, sendo $n$ o número de jogadores de $S$.

A função característica pode ser utilizada para demonstrar a solução dos problemas de distribuição de custos em situações em que a coalizão entre jogadores resulta em economia de custos. Dessa forma, uma condição necessária é a existência de economia causada pela melhor utilização de custos fixos ou pela presença dos efeitos do ganho de escala. A condição necessária pode ser expressa da seguinte forma: $C_{N}\left(d_{1}, d_{2}, \ldots, d_{n}\right)<\sum C_{i}\left(d_{i}\right)$.

Admita-se que o custo total dos serviços prestados pelo departamento centralizador seja de $v(N)=C_{N}\left(d_{1}, d_{2}, \ldots, d_{n}\right)$, e que o custo para cada departamento usuário executar esse mesmo serviço isoladamente seja representado por $v(i)=C_{i}\left(d_{i}\right)$.

A economia de custo pela criação dos departamentos prestadores de serviços internos é dada pela expressão $\sum C_{i}\left(d_{i}\right)-C_{N}\left(d_{1}, d_{2}, \ldots, d_{n}\right)$, que também pode ser entendida como sendo o valor da coalizão. A economia de custos, obtida pela centralização, deve então ser distribuída, e o vetor de distribuição desse ganho é dado por $x=\left(x_{1}, x_{2}, \ldots, x_{n}\right)$.

Suponha-se que exista um departamento que execute atividades de apoio para dois departamentos da empresa; assim sendo, tem-se que $v\left(D_{1}, D_{2}\right)=C_{12}\left(d_{1}, d_{2}\right)$, e o custo de cada departamento usuário é representado 
por $v\left(D_{1}\right)=C_{1}\left(d_{1}\right)$ e $v\left(D_{2}\right)=C_{2}\left(d_{2}\right)$. Dessa forma, a economia de custo da centralização das atividades é dada por $C_{1}\left(d_{1}\right)+C_{2}\left(d_{2}\right)-C_{12}\left(d_{1}, d_{2}\right)$.

É razoável imaginar que, em situações envolvendo a distribuição de custos, os gestores de departamento, agindo como agentes econômicos racionais, irão repartir a economia de custos conseguida pela coalizão. Assim, a utilização da função característica conduz aos conceitos existentes na teoria dos jogos para se obter a "solução do jogo". Para Von Neumann e Morgenstern (1944), a solução para o jogo não era única, mas composta por um conjunto de imputações estáveis, um vetor de valores aceitáveis para cada um dos jogadores participantes da coalizão. Porém, esse tipo de solução não é interessante na distribuição dos ganhos obtidos pela economia de custos, pois não determina os valores a serem ressarcidos pelos departamentos usuários do departamento centralizador.

\subsection{Valor de Shapley}

O conceito de valor neste caso não deve ser confundido com o conceito de valor apregoado pela economia. Refere-se a uma solução dos jogos na forma coalizional que aponta um único vetor de distribuição do ganho, chamado de valor ou valor do jogo para cada jogador. Um dos principais conceitos de valor foi proposto por Shapley (1953), sobre o qual já foram desenvolvidos diversos aperfeiçoamentos para ajustar esses conceitos aos mais diferentes "jogos" da vida real. Pode-se obter maiores detalhes em Roth (1988).

$\mathrm{O}$ valor representa a divisão do resultado do jogo entre os diferentes jogadores que compõem uma coalizão. $\mathrm{O}$ paradigma para se definir a divisão é que ela esteja diretamente associada ao poder dos diferentes jogadores, sendo o poder de um jogador medido pela sua contribuição potencial na coalizão com os demais jogadores.

Isso significa que, caso se trate de distribuição de custos, por exemplo, aquele departamento que mais diminui o consumo de um recurso (diminui o custo da coalizão) deve receber a maior parcela do benefício pela economia.

Segundo Eichberger (1993), o conceito de valor apresentado por Shapley (1953) baseia-se em alguns conceitos avançados, chamados de Axiomas. E para a sugestão da repartição do ganho, produzida pelo valor de Shapley, ser aceita, ela deve obedecer a tais axiomas. Para Eichberger (1993), o que o surpreende na proposição de Shapley (1953) é o pequeno número de requisitos que, reunidos, formam a condição suficiente para definir uma repartição única (o valor ou valor de Shapley) para um jogo na forma coalizional.

Identifica-se por $\phi(v) \equiv\left(\phi_{1}(v), \phi_{2}(v), \phi_{3}(v), \ldots, \phi_{n}(v)\right)$ a função que define a repartição dos ganhos entre os $n$ jogadores de um jogo na forma coalizional $\Gamma=(N, v)$. Cada uma das alocações $\phi(v)$ segue os três axiomas definidos por Shapley (1953): simetria, eficiência e aditividade.
A simetria pode ser entendida por um exemplo simples. Dados dois jogadores $i, j \in N$ que contribuem da mesma forma para a coalizão $S$, então tem-se $v(S \cup\{i\})=v(S \cup\{j\})$ para todo $S \in 2^{N}$, o que indica que $\phi_{i}(v)=\phi_{j}(v)$. Nesse axioma, Shapley determina que todo jogador que possui a mesma contribuição potencial (contribuição marginal) na coalizão de que participa deve receber a mesma quantia na repartição do resultado do jogo.

$\mathrm{O}$ axioma da eficiência afirma que a função de repartição dos resultados $\phi(v)$ distribui o ganho total do jogo, ou seja: $\sum_{i \in C} \phi_{i}(v)=v(C)$. Esse axioma definido por Shapley (1953) refere-se ao conceito de Pareto Eficiente, que rejeita qualquer alocação que proporcione oportunidade de melhoria para um jogador sem perda para um outro.

No axioma da eficiência, está incorporada a definição do jogador dummy. Todavia, antes de se definir esse jogador, é importante conhecer o conceito de carrier. Para um jogo na forma coalizional $\Gamma=(N, v)$, o carrier é a coalizão $C \in 2^{N}$, tal que, para todo $S \in 2^{N}, v(S)=v(S \cap C)$.

Assim, o carrier pode ser descrito como o grupo de jogadores com habilidade (recursos, capital, tempo etc.) de gerar o ganho ou adicionar valor à coalizão. Os jogadores que estão fora do carrier e pertencem à coalizão $S$ não possuem influência sobre o resultado do jogo, já que não contribuem em nada para a coalizão. Um jogador é denominado de dummy quando não contribui para alterar o valor da coalizão. Sendo assim, esse jogador, no momento da distribuição (que considera a contribuição marginal de cada jogador no ganho proporcionado pela coalizão), acaba não recebendo qualquer valor. Se $v(S \cup\{i\})-v(S)=0$, então $\phi_{i}(v)=0$. É como se um departamento não consumisse um tipo de serviço interno e, por isso, não receberia o custo da prestação desse serviço.

Por fim, tem-se o axioma da aditividade, que Shapley em seu artigo denominou de "Lei da Agregação". Quando dois jogos independentes são combinados, seus resultados precisam ser somados jogador a jogador. Se $v$ e $u$ são funções características, então $\phi(v+u)=\phi(v)+\phi(u)$. Este último axioma de Shapley não é uma suposição trivial. $\mathrm{O}$ axioma da aditividade refere-se à seguinte situação: se um valor for escolhido para dois jogos realizados simultaneamente pelos mesmos jogadores, este deve ser o valor a ser conseguido se esses dois jogos forem realizados em momentos distintos. E se $v$ e $u$ são funções características desses jogos, então esse valor é igual a $v+u$.

Considerando-se os axiomas supra descritos, de acordo com o trabalho de Shapley (1953), conclui-se que existe apenas um único vetor de repartição (valor dos jogadores) que atende aos conceitos de eficiência, simetria, aditividade e do jogador dummy, vetor esse fornecido pelo valor de Shapley. A prova desse teorema pode ser vista em Shapley (1953) ou em Burger (1963). 
As propriedades de simetria e do jogador dummy garantem o caráter de justiça na distribuição do resultado do jogo. A eficiência indica que todo o valor do jogo será repartido entre os jogadores da coalizão, enquanto que a aditividade permite presumir que esse valor é único. Isso porque, independentemente de como irão jogar os componentes da coalizão, conseguirão apenas a quantia que poderia ser alcançada em um único jogo.

Admitindo as características apresentadas, o valor de Shapley é dado pela Equação 1:

$$
\phi_{i}(v)=\sum_{\substack{S \subset N \\ i \in S}} \frac{(|S|-1) !(n-|S|) !}{n !}[v(S)-v(S-\{i\})]
$$

A primeira parte da fórmula representa a probabilidade de um jogador em particular participar, em diversos momentos distintos, de coalizões com $S$ participantes. A probabilidade do jogador $i$ encontrar a coalizão $S-\{i\}$ já formada é igual a $\frac{(|S|-1) !(n-|S|) !}{n !}$. O denominador representa o número total de permutações com $n$ jogadores. $\mathrm{O}$ numerador representa o número dessas permutações em que $|S|-1$ jogadores de $S-\{i\}$ vêm primeiro (são $(|S|-1)$ ! maneiras), após vem o jogador $i$, e só depois entram os demais jogadores $n-|S|$ (são $(n-|S|)$ ! maneiras).

A segunda parte da equação é denominada de contribuição marginal do jogador $i$ (Equação 2):

$$
C M_{i}=[v(S)-v(S-\{i\})]
$$

Sendo $S-\{i\}$ a coalizão dos jogadores de $S$ que não contém $i$, e $S$ a coalizão formada por todos os jogadores de $S$ incluindo $i$, a contribuição (custo ou lucro) marginal $C M_{i}$ de inclusão do jogador $i$ na coalizão $S$ é dada por $[v(S)-v(S-\{i\})]$

Assim, o valor de Shapley $\left(\phi_{i}(v)\right)$ nada mais é do que a média de contribuição marginal do jogador $i$ para a coalizão $S$, considerando que os jogadores formem essa coalizão em uma sequência aleatória.

\subsection{Custo marginal e aleatoriedade segundo Shapley}

No caso da alocação de custos, o valor de Shapley pode ser explicado como sendo a expectativa do ganho marginal adicionado pelo departamento que entra na coalizão, dado que as ordens de entrada nas diversas coalizões possíveis para o usuário são igualmente prováveis. Para explicar o conceito de custo marginal dentro do contexto da alocação de custo pelo valor de Shapley, lança-se mão de um exemplo apresentado por Jensen (1977).

Nesse exemplo, o autor (ibid.) descreve uma situação em que dois usuários possuem demandas específicas de determinado serviço, definidas por $k_{1}=200 \mathrm{e} k_{2}=1.000$ unidades.
O custo mínimo de disponibilização dessas unidades para o primeiro usuário é de $C\left(k_{1}\right)=\$ 400 \mathrm{e}$, para o segundo, de $C\left(k_{2}\right)=\$ 900$. Por outro lado, se os dois compartilhassem uma mesma estrutura que disponibilizasse o serviço demandado pelos dois departamentos $(K=1.200)$, o custo mínimo seria de $C(K)=\$ 1.000$.

Admita-se que os dois usuários estão de acordo com as condições básicas de alocação de custos (descritas no item anterior) definidas pelo valor de Shapley. Nesse sentido, ainda segundo o mesmo autor (ibid.):

Os axiomas conduzem a uma alocação baseada no custo incremental. [...] o custo marginal é a diferença entre dois recursos hipotéticos - um capaz de satisfazer qualquer subconjunto de demandas e outro que satisfaz, em adição, a demanda de um usuário em particular. Obviamente, o custo incremental varia com as alterações nos subconjuntos de demandas que o novo usuário aumenta. (SHUBIK, 1962).

No caso descrito, como já comentado, as necessidades dos dois usuários podem ser cobertas de duas formas: ou na compra de recursos específicos por usuário, ou na compra de uma estrutura conjunta capaz de atender às necessidades simultaneamente.

Assim, o primeiro usuário pode ter suas necessidades atendidas pela compra de um recurso específico por $\$ 400$ ou por uma estrutura comum por $\$ 1.000$. O custo incremental de satisfazer as necessidades do primeiro usuário, se ele entrar na coalizão após o segundo usuário, pela estrutura comum é de $\$ 100(\$ 1.000-\$ 900)$, pois o segundo usuário custou na coalizão $\$ 900$. Ou seja, o custo incremental é o acréscimo no custo causado pela entrada da demanda do primeiro usuário no subconjunto formado pela demanda do segundo usuário.

$\mathrm{O}$ custo incremental para atender à demanda do primeiro usuário com uma estrutura específica é de $\$ 400$ (\$ $400-0)$, que representa a entrada do primeiro usuário em um subconjunto sem demanda, o que resulta no próprio custo do primeiro usuário. Da mesma forma, o custo incremental para atender às necessidades do segundo usuário, supondo que o segundo usuário entre na coalizão após o primeiro usuário, é igual a \$ $600(\$ 1.000$ - \$400) em uma estrutura conjunta e $\$ 900(\$ 900-0)$ em uma estrutura específica.

Se os dois usuários decidirem construir uma estrutura conjunta e concordarem em alocar os custos comuns de forma que os quatro axiomas sejam satisfeitos, então, a seguinte distribuição de custos será aceita por esses usuários: $\alpha=(250,750)$.

Sendo assim, uma forma de descrever o custo total de uma estrutura comum é pelo incremento causado de acordo com a entrada de cada usuário em uma sequência pré-definida. 
Por exemplo, o custo total da estrutura que atenderá os dois usuários é de \$1.000, que pode ser explicado como necessário para atender às necessidades do primeiro usuário, (\$400), mais um custo adicional para satisfazer às necessidades do segundo usuário $(\$ 600)$ ou, apenas alterando a ordem de entrada dos usuários na coalizão, os \$ 1.000 podem ser justificados para atender às necessidade do segundo usuário (\$900), mais um custo adicional para atender à demanda do primeiro usuário (\$100).

Como se pôde observar, a decomposição do custo total depende da sequência em que as demandas são reconhecidas. Nesse caso, existem duas sequências possíveis: $(1,2)$ e $(2,1)$, no caso de três usuários, seis, e no caso de $n$, tem-se $n$ ! sequências. O custo marginal é sensível às diversas alternativas de caminhos possíveis (e que dependem do número de usuários envolvidos). Cada usuário terá um custo marginal calculado para cada caminho alternativo possível. A alocação de custo sugerida pela utilização do valor de Shapley e pelos axiomas definidos por Loehman e Whinston (1971) corresponde, simplesmente, à soma dos custos marginais dos diversos caminhos possíveis dividida pelo número de alternativas. Ou seja, corresponde ao custo médio incremental.

\subsection{Valor de Shapley e a alocação de custos}

O valor de Shapley é um método pelo qual os jogadores têm conhecimento a priori dos benefícios esperados de se entrar em um jogo. Para demonstrar o relacionamento do valor de Shapley com os problemas de alocação de custos, utiliza-se, especificamente, a alocação de custos de departamentos prestadores de serviços internos. Para isso, admita-se que o custo total de um departamento de serviço interno (departamento de manutenção ou departamento de TI) precise ser alocado entre $n$ departamentos, denominados por $N=(1,2, \ldots, n)$. A função $v(S)$ descreve o ganho total obtido pela coalizão $S$ quando todos esses departamentos cooperam para assegurar a maior eficiência na disponibilização do serviço comum.

O ganho nesse jogo pode ser entendido como a redução de custo da estrutura que suporta o serviço comum, e também como sendo passível de transferência entre os jogadores participantes da coalizão. Essa transferência é chamada de transferência de utilidade e ocorre, nesse caso, pela distribuição da economia de custo trazida por um dos jogadores entre todos os outros da coalizão. Desse modo, a medida da utilidade do tomador de decisão, neste trabalho, é dada pela comparação entre o custo atribuído em função do jogo e o custo individual do departamento, considerando que ele não venha a fazer parte da coalizão.

Os tomadores de decisão (gestores dos departamentos) estarão de acordo com as regras do jogo (de distribuição de custos comuns) se preferirem o custo alocado pelo jogo ao invés do custo de construção da estrutura do serviço interno de forma isolada dos demais departa- mentos. O valor de Shapley para um departamento $i$ é dado por (Equação 3):

$$
D_{i}=\sum_{\substack{S \subset N \\ i \in S}} \frac{(s-1) !(n-s) !}{n !}[v(S)-v(S-\{i\})]
$$

Inicialmente, para se calcular o vetor de distribuição de custos utilizando o valor de Shapley, é necessário saber que $s$ é o número de departamentos na coalizão $S$ e $n$ é o número total de departamentos. Para demonstrar como se pode aplicar o conceito do valor de Shapley em um jogo de alocação de custos, admita-se a existência de três departamentos usuários, ou $N=(A, B, C)$.

A empresa XPTO deseja diminuir seus gastos com informática e, para isso, avalia a criação de um departamento de processamento de dados para atender aos principais departamentos operacionais da empresa. Atualmente, os principais sistemas transacionais da empresa estão sendo processados por servidores e os demais recursos mantidos pelos próprios departamentos operacionais. Para atingir o objetivo de centralização das atividades de TI, a empresa precisa deixar clara para os departamentos usuários a vantagem obtida em termos de custos e de melhoria do resultado dos departamentos.

$\mathrm{O}$ primeiro passo dado pela empresa foi o cálculo estimado do consumo de recursos de TI que os três departamentos operacionais demandariam do novo departamento de processamento de dados. A empresa soube, por meio desse levantamento, que os departamentos A e C consomem uma quantidade de recursos de TI (medidos em MIPS - Milhões de Instruções por Segundo) menor (1.733 MIPS e 1.333 MIPS, respectivamente) do que o departamento B, que consome mais da metade do que é consumido pelos três departamentos (3.933 MIPS).

Realizadas as estimativas dos custos, o problema passa a ser de avaliação do investimento. A empresa avalia se, considerando a economia obtida, vale a pena ou não a centralização dos serviços de TI. Caso seja interessante para a empresa, o problema passa a ser convencer os departamentos operacionais de que a centralização também trará benefícios maiores do que a manutenção de estruturas próprias de processamento de dados.

Dando sequência à apresentação do exemplo, o próximo passo é a obtenção de estimativas confiáveis para as diversas situações possíveis:

a) o departamento A mantém sua estrutura de TI por $\$ 5.000,00$;

b) o departamento B mantém sua estrutura de TI por $\$ 3.000,00$;

c) o departamento C mantém sua estrutura de TI por $\$ 5.000,00$;

d) se apenas A e B cooperassem entre si para montar uma estrutura comum, isso resultaria em um custo de $\$ 6.000,00$; 
e) se B e C entrassem em um acordo, isso resultaria em um custo de $\$ 7.000,00$;

f) se $\mathrm{A}$ e $\mathrm{C}$ cooperassem, seria alcançado um custo de $\$ 10.000,00$; e

g) se A, B e C utilizassem a mesma estrutura de TI, seria alcançado um custo de $\$ 10.500,00$.

É fácil perceber que a melhor solução para atender às necessidades dos três departamentos (e que representa o menor custo para a empresa) é a criação de uma unidade centralizadora do serviço, comum aos três departamentos operacionais e, nesse caso, a empresa economizaria cerca de $\$ 2.500,00$. Todavia, os departamentos operacionais precisam concordar com a forma como os custos serão alocados para os departamentos.

Uma maneira comum de alocar os custos entre os departamentos é pelo critério da utilização do serviço. Nesse caso, o custo de cada um dos departamentos é dado pela proporção de seu consumo em relação ao consumo total dos três departamentos (nesse exemplo, medido em "minutos de processamento"). Pelo critério da utilização, a distribuição resultaria no seguinte vetor de custos: $\alpha=(2.600,2.000,5.900)$.

Essa distribuição de custos não é vantajosa para o departamento C, que receberá uma conta de $\$ 5.900,00$ $(\$ 10.500,00 / 7.000$ MIPS $* 3.933$ MIPS $=\$ 5.900,00) . \mathrm{O}$ departamento $\mathrm{C}$ sabe que, sozinho, mantém uma estrutura de TI que custa $\$ 5.000,00$. Embora $\mathrm{C}$ tenha contribuído para que a empresa obtivesse economia de custo, pela melhor utilização de custos fixos, não se beneficia dessa economia pelo critério de distribuição escolhido pela empresa.

Outra forma de alocar esse custo é considerar o custo marginal médio de cada departamento nas diversas coalizões possíveis. Para tal, Loehman e Whinston (1971) afirmam que é importante que a empresa negocie antecipadamente as "condições básicas" (axiomas) sobre quais alocações de custos serão feitas entre os usuários do serviço, sendo elas:

a) o custo a ser alocado é igual ao custo da prestação de serviço aos usuários;

b) a alocação de custos será realizada exclusivamente com base na contribuição marginal causada pelo usuário, não com base na contribuição marginal de outros usuários;

c) os usuários com contribuições idênticas receberão custos iguais; e

c) a alocação de custos será homogênea com grau um, ou seja, se o custo incremental de um usuário elevar-se em um certo percentual, a alocação de custos para esse usuário será elevada pelo mesmo percentual;

Loehman e Whinston (1971) provaram que só existe um vetor de alocação de custos que obedece a esses quatro axiomas, e é obtido pela utilização da função definida em Shapley (1953). Sendo assim, qual seria o custo alocado para cada um dos departamentos usuários? Utilizando-se a fórmula desenvolvida por Shapley, a distribuição de custos seria realizada da seguinte forma: $\alpha=(4.166,67$, $1.666,67,4.666,67)$.

Se observarmos o vetor de alocação produzido pelo valor de Shapley, não há um valor que seja maior do que cada um pagaria se resolvesse não entrar na coalizão. $\mathrm{Se}$ os departamentos assumissem um comportamento guiado pelos critérios racionais de alocação dos custos por departamento, o vetor de alocação de custos calculado pelo valor de Shapley seria um instrumento ideal para alocação desse custo por departamento (ROTH; VERRECCHIA, 1979).

\section{Considerações finais}

O modelo apresentado neste trabalho, em função das características de modelagem dos jogos cooperativos e, em especial, do valor proporcionado pela utilização da fórmula de solução de um jogo cooperativo desenvolvida por Shapley (1953), produz um vetor de alocação de custos que possui aspectos semelhantes a uma negociação entre os departamentos envolvidos na alocação de custos. Por outro lado, minimiza o tempo que poderia ser gasto com a negociação de taxas de alocação de custos.

No entanto, devem-se considerar alguns fatores que dificultam a utilização deste modelo, como, por exemplo: alguns dos axiomas podem ser explicados facilmente para os usuários (axiomas 1, 3 e 4, por exemplo), contudo, outros precisam ser explicados com maior grau de profundidade (axioma 2) ou transformados em situações que permitam fácil assimilação dos critérios por parte dos usuários. Além disso, o modelo pressupõe que se conheçam, a priori, os custos individuais dos jogadores e também de cada uma das coalizões possíveis no jogo. Isso pode fazer com que o modelo se torne complexo caso haja um número grande de jogadores. Pesquisas posteriores podem se utilizar destes problemas para elaboração de modelos otimizados de alocação de custos.

O que se apresentou neste trabalho é uma das metodologias para se alocar custos. O ponto realmente importante é o entendimento dos resultados produzidos pelos critérios utilizados na modelagem do sistema de alocação de custos pelo valor de Shapley, e se esses critérios ajustam-se aos objetivos que a empresa pretende atingir com os números produzidos pela alocação. Acredita-se que o valor de Shapley produz uma informação de alocação de custos que carrega nela características que poderiam ser utilizadas para avaliação de performance dos departamentos usuários, bem como um instrumento de controle dos custos dos departamentos de serviços internos. 


\title{
Analysis and characterization of cost models that use Shapley value for cost allocation within departments
}

\begin{abstract}
In order to obtain benefits (economies of scale and scope), several organizations create service provider structures such as the computer departments. The distribution of the service rendering costs among the departments involved, called the first stage cost allocation, promotes the cost control and encourages an efficient use of resources. This study shows the characteristics of the cost allocation model of a service rendering based on the concepts of the Cooperative Game Theory. Shapley value was used in order to find the cooperative game solution. This work indicates that this math model of gain distribution (economies of scale and scope) generates, under the rational decision maker's view, the best cost distribution results. This is due to the fact they are based on the marginal cost of insertion of each of the service users. Regarding methodology, this research can be classified as exploratory due to its scope and bibliography.
\end{abstract}

Keywords: Cost allocation. Cooperative game theory. Shapley value.

\section{Referências bibliográficas}

BAILEY, E. E.; FRIEDLAENDER, A. F. Market Structure and Multiproduct Industries. Journal of Economic Literature, v. 20, n. 3, p. 1024-1048, Sep. 1982.

BALACHANDRAN, B. V.; RAMAKRISHNAN, R. T. S. Joint Cost Allocation: A unified Approach. The Accounting Review, v. 56, n. 1, p. 85-96, Jan. 1981.

BURGER, E. Introduction to Theory of Games. N.J: Prentice Hall Inc., 1963.

BUTLER, M.; WILLIAMS, H. P. Fairness Versus Efficiency in Charging for the Use of Common Facilities. Journal of Operational Research Society, v. 53, p. 1324-1329, 2002.

CALLEN, J. L. Financial Cost Allocations: A Game Theoretic Approach. The Accounting Review, v. 53, n. 2, p.303-308, Apr. 1978.

DRURY, C. Management and Cost Accounting. 6 ed. [S.L.]: Thompson, 2004.

EICHBERGER, J. Game Theory for Economists. London: Academic Press Inc., 1993.

GIL, A. C. Como Elaborar Projetos de Pesquisa. São Paulo: Atlas, 1987. $159 \mathrm{p}$.

HAMLEN, S. S.; HAMLEN, W. A.; TSCHIRHART, J. T. The use of Core Theory in Evaluating Joint Cost Allocation Schemes. The Accounting Review, v. 52, n. 3, p. 616-627, July 1977.

HORNGREEN, C. T. Contabilidade de Custos: Um enfoque Administrativo. São Paulo: Atlas, 1986. 1077 p.

JENSEN, D. L. A Class of Mutually Satisfactory Allocations. The Accounting Review, v. 52, n. 4, p. 842-856, Oct. 1977.
KAPLAN, R. S.; ATKINSON, A. A. Advanced Management Accounting. 3 ed. New Jersey: Prentice Hall Inc., 1998. 798 p.

LEIKAM, S. L. An Evaluation of Activity-Based Costing and Functional Based Costing: A Game Theoretic Approach. Oklahoma, 2000. 168f. Thesis (Doctor of Philosophy) - Faculty of The Graduate College, Oklahoma State University.

LOEHMAN, E.; WHINSTON, A. A New Theory of Pricing and Decision-Making for Public Investment. The Bell Journal of Economics and Management Science, v. 2, n. 2, p. 606-625, 1971.

PANZAR, J. C.; WILLIG, R. D. Economies of Scope. The American Economic Review, v. 22, n. 2, p. 268-272, May 1981.

ROTH, A. E. The Shapley Value: Essays in honor of Lloyd S. Shapley. Cambridge: Cambridge University Press, 1988.

ROTH, A. E.; VERRECCHIA, R. E. The Shapley Value as Applied to Cost Allocation: A Reinterpretation. The Accounting Review, v. 17, n. 1, p. 295-303, 1979.

SHAPLEY, L. S. A Value for n-Person Games. In: KUHN, H. W.; TUCKER, A. W. (Eds.). Contribution to the Theory of Games. Annals of Mathematics Studies. New Jersey: Princeton University Press, 1953. p. 307-317.

SHUBIK, M. Incentives, decentralized control, the assignment of joint cost and internal pricing. Management Science, v. 8, n. 3, p. 325-343, Apr. 1962.

VON NEWMAN, J.; MORGENSTERN, O. Theory of games and economic behavior. Princeton: Princeton University Press, 1944.

ZIMMERMAN, J. L. Accounting for Decision Making and Control. 4 ed. Boston: McGraw Hill, 2003. 


\section{Sobre os autores}

\section{Francisco Antonio Bezerra}

\section{Jefferson Fernando Grande}

\section{Adriano José da Silva}

Programa de Pós-Graduação em Ciências Contábeis - PPGCC/FURB,

Fundação Universidade Regional de Blumenau - FURB,

PPGCC - Campus I, sala D-202, Rua Antônio da Veiga, 140 - CP 1507, CEP 89012-900, Victor Konder, Blumenau, SC, Brasil,

e-mails: fbezerra@furb.br, jeffersonfg@ig.com.br, ajs@al.furb.br

Recebido em 28/9/2007

Aceito em 21/1/2009 